PROCEEDINGS OF THE

AMERICAN MATHEMATICAL SOCIETY

Volume 131, Number 8, Pages 2453-2457

S 0002-9939(02)06775-8

Article electronically published on November 13, 2002

\title{
TRANSFINITE SEQUENCES OF CONTINUOUS AND BAIRE CLASS 1 FUNCTIONS
}

\author{
MÁRTON ELEKES AND KENNETH KUNEN \\ (Communicated by Alan Dow)
}

\begin{abstract}
The set of continuous or Baire class 1 functions defined on a metric space $X$ is endowed with the natural pointwise partial order. We investigate how the possible lengths of well-ordered monotone sequences (with respect to this order) depend on the space $X$.
\end{abstract}

\section{INTRODUCTION}

Any set $\mathcal{F}$ of real valued functions defined on an arbitrary set $X$ is partially ordered by the pointwise order; that is, $f \leq g$ iff $f(x) \leq g(x)$ for all $x \in X$. Then, $f<g$ iff $f \leq g$ and $g \not \leq f$; equivalently, $f(x) \leq g(x)$ for all $x \in X$ and $f(x)<g(x)$ for at least one $x \in X$. Our aim will be to investigate the possible lengths of the increasing or decreasing well-ordered sequences of functions in $\mathcal{F}$ with respect to this order. A classical theorem (see Kuratowski [7], §24.III, Theorem 2') asserts that if $\mathcal{F}$ is the set of Baire class 1 functions (that is, pointwise limits of continuous functions) defined on a Polish space $X$ (that is, a complete separable metric space), then there exists a monotone sequence of length $\xi$ in $\mathcal{F}$ iff $\xi<\omega_{1}$. P. Komjáth [5] proved that the corresponding question concerning Baire class $\alpha$ functions for $2 \leq \alpha<\omega_{1}$ is independent of $Z F C$.

In the present paper we investigate what happens if we replace the Polish space $X$ by an arbitrary metric space.

Section 1 considers chains of continuous functions. We show that for any metric space $X$, there exists a chain in $C(X, \mathbb{R})$ of order type $\xi$ iff $|\xi| \leq d(X)$. Here, $|A|$ denotes the cardinality of the set $A$, while $d(X)$ denotes the density of the space $X$, that is,

$$
d(X)=\max (\min \{|D|: D \subseteq X \& \bar{D}=X\}, \omega) .
$$

In particular, for separable $X$, every well-ordered chain has countable length, just as for Polish spaces.

Section 2 considers chains of Baire class 1 functions on separable metric spaces. Here, the situation is entirely different from the case of Polish spaces, since on some

Received by the editors December 11, 2001 and, in revised form, March 13, 2002.

2000 Mathematics Subject Classification. Primary 26A21; Secondary 03E17, 54C30.

Key words and phrases. Baire class 1, separable metric space, transfinite sequence of functions. The second author was partially supported by NSF Grant DMS-0097881. 
separable metric spaces there are well-ordered chains of every order type less than $\omega_{2}$. Furthermore, the existence of chains of type $\omega_{2}$ and longer is independent of $Z F C+\neg C H$. Under $M A$, there are chains of all types less than $\mathfrak{c}^{+}$, whereas in the Cohen model, all chains have type less than $\omega_{2}$.

We note here that instead of examining well-ordered sequences, which is a classical problem, we could try to characterize all the possible order types of linearly ordered subsets of the partially ordered set $\mathcal{F}$. This problem was posed by M. Laczkovich, and is considered in detail in [3].

\section{Sequences of COntinuous functions}

Lemma 1.1. For any topological space $X$ : If there is a well-ordered sequence of length $\xi$ in $C(X, \mathbb{R})$, then $\xi<d(X)^{+}$.

Proof. Let $\left\{f_{\alpha}: \alpha<\xi\right\}$ be an increasing sequence in $C(X, \mathbb{R})$, and let $D \subseteq X$ be a dense subset of $X$ such that $d(X)=\max (|D|, \omega)$. By continuity, the $f_{\alpha}\lceil D$ are all distinct; so, for each $\alpha<\xi$, choose a $d_{\alpha} \in D$ such that $f_{\alpha}\left(d_{\alpha}\right)<f_{\alpha+1}\left(d_{\alpha}\right)$. For each $d \in D$ the set $E_{d}=\left\{\alpha: d_{\alpha}=d\right\}$ is countable, because every well-ordered subset of $\mathbb{R}$ is countable. Since $\xi=\bigcup_{d \in D} E_{d}$, we have $|\xi| \leq \max (|D|, \omega)=d(X)$.

The converse implication is not true in general. For example, if $X$ has the countable chain condition (ccc), then every well-ordered chain in $C(X, \mathbb{R})$ is countable (because $X \times \mathbb{R}$ is also ccc). However, the converse is true for metric spaces:

Lemma 1.2. If $(X, \varrho)$ is any non-empty metric space and $\prec$ is any total order of the cardinal $d(X)$, then there is a chain in $C(X, \mathbb{R})$ which is isomorphic to $\prec$.

Proof. First, note that every countable total order is embeddable in $\mathbb{R}$, so if $d(X)=$ $\omega$, then the result follows trivially using constant functions. In particular, we may assume that $X$ is infinite, and then fix $D \subseteq X$ which is dense and of size $d(X)$. For each $n \in \omega$, let $D_{n}$ be a subset of $D$ which is maximal with respect to the property $\forall d, e \in D_{n}\left[d \neq e \rightarrow \varrho(d, e) \geq 2^{2-n}\right]$. Then $\bigcup_{n} D_{n}$ is also dense, so we may assume that $\bigcup_{n} D_{n}=D$. We may also assume that $\prec$ is a total order of the set $D$. Now, we shall produce $f_{d} \in C(X, \mathbb{R})$ for $d \in D$ such that $f_{d}<f_{e}$ whenever $d \prec e$.

For each $n$, if $c \in D_{n}$, define $\varphi_{c}^{n}(x)=\max \left(0,2^{-n}-\varrho(x, c)\right)$. For each $d \in D$, let $\psi_{d}^{n}=\sum\left\{\varphi_{c}^{n}: c \in D_{n} \& c \prec d\right\}$. Since every $x \in X$ has a neighborhood on which all but at most one of the $\varphi_{c}^{n}$ vanish, we have $\psi_{d}^{n} \in C\left(X,\left[0,2^{-n}\right]\right)$, and $\psi_{d}^{n} \leq \psi_{e}^{n}$ whenever $d \prec e$. Thus, if we let $f_{d}=\sum_{n<\omega} \psi_{d}^{n}$, we have $f_{d} \in C(X,[0,2])$, and $f_{d} \leq$ $f_{e}$ whenever $d \prec e$. But also, if $d \in D_{n}$ and $d \prec e$, then $\psi_{d}^{n}(d)=0<2^{-n}=\psi_{e}^{n}(d)$, so actually $f_{d}<f_{e}$ whenever $d \prec e$.

Putting these lemmas together, we have:

Theorem 1.3. Let $(X, \varrho)$ be a metric space. Then there exists a well-ordered sequence of length $\xi$ in $C(X, \mathbb{R})$ iff $\xi<d(X)^{+}$.

Corollary 1.4. A metric space $(X, \varrho)$ is separable iff every well-ordered sequence in $C(X, \mathbb{R})$ is countable.

\section{Sequences of Baire Class 1 Functions}

If we replace continuous functions by Baire class 1 functions, then Corollary 1.4 becomes false, since on some separable metric spaces we can get well-ordered sequences of every type less than $\omega_{2}$. To prove this, we shall apply some basic facts 
about $\subset^{*}$ on $\mathcal{P}(\omega)$. As usual, for $x, y \subseteq \omega$, we say that $x \subseteq^{*} y$ iff $x \backslash y$ is finite. Then $x \subset^{*} y$ iff $x \backslash y$ is finite and $y \backslash x$ is infinite. This $\subset^{*}$ partially orders $\mathcal{P}(\omega)$.

Lemma 2.1. If $X \subset \mathcal{P}(\omega)$ is a chain in the order $\subset^{*}$, then on $X$ (viewed as a subset of the Cantor set $\left.2^{\omega} \cong \mathcal{P}(\omega)\right)$ there is a chain of Baire class 1 functions which is isomorphic to $\left(X, \subset^{*}\right)$.

Proof. Note that for each $x \in X$,

$$
\left\{y \in X: y \subseteq^{*} x\right\}=\bigcup_{m \in \omega}\{y \in X: \forall n \geq m[y(n) \leq x(n)]\},
$$

which is an $F_{\sigma}$ set in $X$. Likewise, the sets $\left\{y \in X: y \supseteq^{*} x\right\},\left\{y \in X: y \subset^{*} x\right\}$, and $\left\{y \in X: y \supset^{*} x\right\}$, are all $F_{\sigma}$ sets in $X$, and hence also $G_{\delta}$ sets. It follows that if $f_{x}: X \rightarrow\{0,1\}$ is the characteristic function of $\left\{y \in X: y \subset^{*} x\right\}$, then $f_{x}: X \rightarrow \mathbb{R}$ is a Baire class 1 function. Then $\left\{f_{x}: x \in X\right\}$ is the required chain.

Lemma 2.2. For any infinite cardinal $\kappa$, suppose that $\left(\mathcal{P}(\omega), \subset^{*}\right)$ contains a chain $\left\{x_{\alpha}: \alpha<\kappa\right\}$ (i.e., $\left.\alpha<\beta \rightarrow x_{\alpha} \subset^{*} x_{\beta}\right\}$ ). Then $\left(\mathcal{P}(\omega), \subset^{*}\right)$ contains a chain $X$ of size $\kappa$ such that every ordinal $\xi<\kappa^{+}$is embeddable into $X$.

Proof. Let $S=\bigcup_{1 \leq n<\omega} \kappa^{n}$. For $s=\left(\alpha_{1}, \ldots, \alpha_{n-1}, \alpha_{n}\right) \in S$, let

$$
s^{+}=\left(\alpha_{1}, \ldots, \alpha_{n-1}, \alpha_{n}+1\right) \text {. }
$$

Starting with the $x_{(\alpha)}=x_{\alpha}$, choose $x_{s} \in \mathcal{P}(\omega)$ by induction on length $(s)$ so that

$$
x_{s}=x_{s \frown 0} \subset^{*} x_{s \frown \alpha} \subset^{*} x_{s \frown \beta} \subset^{*} x_{s^{+}}
$$

whenever $s \in S$ and $0<\alpha<\beta<\kappa$. Let $X=\left\{x_{s}: s \in S\right\}$. Then, whenever $x, y \in$ $X$ with $x \subset^{*} y$, the ordinal $\kappa$ is embeddable in $(x, y)=\left\{z \in X: x \subset^{*} z \subset^{*} y\right\}$. From this, one easily proves by induction on $\xi<\kappa^{+}$(using $\left.\operatorname{cf}(\xi) \leq \kappa\right)$ that $\xi$ is embeddable in each such interval $(x, y)$.

Since $\mathcal{P}(\omega)$ certainly contains a chain of type $\omega_{1}$, these two lemmas yield:

Theorem 2.3. There is a separable metric space $X$ on which, for every $\xi<\omega_{2}$, there is a well-ordered chain of length $\xi$ of Baire class 1 functions.

Under $\mathrm{CH}$, this is best possible, since there will be only $2^{\omega}=\omega_{1}$ Baire class 1 functions on a separable metric space, so there could not be a chain of length $\omega_{2}$. Under $\neg C H$, the existence of longer chains of Baire class 1 functions depends on the model of set theory. It is consistent with $\mathfrak{c}=2^{\omega}$ being arbitrarily large that there is a chain in $\left(\mathcal{P}(\omega), \subset^{*}\right)$ of type $\mathfrak{c}$; for example, this is true under $M A$ (see [2]). In this case, there will be a separable $X$ with well-ordered chains of all lengths less than $\mathfrak{c}^{+}$. However, in the Cohen model, where $\mathfrak{c}$ can also be made arbitrarily large, we never get chains of type $\omega_{2}$. We shall prove this by using the following lemma, which relates it to the rectangle problem:

Lemma 2.4. Suppose that there is a separable metric space $Y$ with an $\omega_{2}$-chain of Borel subsets, $\left\{B_{\alpha}: \alpha<\omega_{2}\right\}$ (so, $\alpha<\beta \rightarrow B_{\alpha} \varsubsetneqq B_{\beta}$ ). Then in $\omega_{2} \times \omega_{2}$, the well-order relation $<$ is in the $\sigma$-algebra generated by the set of all rectangles, $\left\{S \times T: S, T \in \mathcal{P}\left(\omega_{2}\right)\right\}$. 
Proof. Each $B_{\alpha}$ has some countable Borel rank. Since there are only $\omega_{1}$ ranks, we may, by passing to a subsequence, assume that the ranks are bounded. Say each $B_{\alpha}$ is a $\Sigma_{\mu}^{0}$ set for some fixed $\mu<\omega_{1}$.

Let $J=\omega^{\omega}$, and let $A \subseteq Y \times J$ be a universal $\Sigma_{\mu}^{0}$ set; that is, $A$ is $\Sigma_{\mu}^{0}$ in $Y \times J$ and every $\Sigma_{\mu}^{0}$ subset of $Y$ is of the form $A^{j}=\{y:(y, j) \in A\}$ for some $j \in J$ (see [7], §31). Now, for $\alpha, \beta<\omega_{2}$, fix $y_{\alpha} \in B_{\alpha+1} \backslash B_{\alpha}$, and fix $j_{\beta} \in J$ such that $A^{j_{\beta}}=B_{\beta}$. Then $\alpha<\beta$ iff $\left(y_{\alpha}, j_{\beta}\right) \in A$. Thus, $\left\{\left(y_{\alpha}, j_{\beta}\right): \alpha<\beta<\omega_{2}\right\}$ is a Borel subset of $\left\{y_{\alpha}: \alpha<\omega_{2}\right\} \times\left\{j_{\beta}: \beta<\omega_{2}\right\}$, and is hence in the $\sigma$-algebra generated by open rectangles, so $<$, as a subset of $\omega_{2} \times \omega_{2}$, is in the $\sigma$-algebra generated by rectangles.

Theorem 2.5. Assume that the well-order relation $<$ on $\omega_{2}$ is not in the $\sigma$-algebra generated by the set of all rectangles. Then no separable metric space can have a chain of length $\omega_{2}$ of Baire class 1 functions.

Proof. Suppose that $\left\{f_{\alpha}: \alpha<\omega_{2}\right\}$ is a chain of Baire class one functions on the separable metric space $X$. Let $B_{\alpha}=\left\{(x, r) \in X \times \mathbb{R}: r \leq f_{\alpha}(x)\right\}$. Then the $B_{\alpha}$ form an $\omega_{2}$-chain of Borel subsets of the separable metric space $X \times \mathbb{R}$, so we have a contradiction by Lemma 2.4

Finally, we point out that the hypothesis of this theorem is consistent, since it holds in the extension $V[G]$ formed by adding $\geq \omega_{2}$ Cohen reals to a ground model $V$ which satisfies $\mathrm{CH}$. This fact was first proved in [6]. It also follows from the more general principle $\mathrm{HP}_{2}\left(\omega_{2}\right)$ of Brendle, Fuchino, and Soukup [1. They define this principle, prove that it holds in Cohen extensions (and in a number of other forcing extensions), and show the following:

Lemma 2.6. $H P_{2}(\kappa)$ implies that if $R$ is any relation on $\mathcal{P}(\omega)$ which is first-order definable over $H\left(\omega_{1}\right)$ from a fixed element of $H\left(\omega_{1}\right)$, then there is no $X \subseteq \mathcal{P}(\omega)$ such that $(X ; R)$ is isomorphic to $(\kappa ;<)$.

These matters are also discussed in [4], which indicates how such statements are verified in Cohen extensions. Here, $H\left(\omega_{1}\right)$ denotes the set of hereditarily countable sets.

Lemma 2.7. $H P_{2}\left(\omega_{2}\right)$ implies that in $\omega_{2} \times \omega_{2}$, the well-order relation $<$ is not in the $\sigma$-algebra generated by the set of all rectangles, $\left\{S \times T: S, T \in \mathcal{P}\left(\omega_{2}\right)\right\}$.

Proof. Suppose that $<$ were in this $\sigma$-algebra. Then we would have fixed $K_{n} \subseteq \omega_{2}$ for $n<\omega$ such that $<$ is in the $\sigma$-algebra generated by all the $K_{m} \times K_{n}$. For each $\alpha$, let $u_{\alpha}=\left\{n \in \omega: \alpha \in K_{n}\right\}$. There is then a formula $\varphi(x, y, z)$ and a fixed $w \in H\left(\omega_{1}\right)$ such that for all $\alpha, \beta<\omega_{2}, \quad \alpha<\beta$ iff $H\left(\omega_{1}\right) \models \varphi\left(u_{\alpha}, u_{\beta}, w\right)$; here, $w$ encodes the particular countable boolean combination used to get $<$ from the $K_{n}$. Now, if $X=\left\{u_{\alpha}: \alpha<\omega_{2}\right\}$, then $\varphi$ defines a relation $R$ on $H\left(\omega_{1}\right)$ such that $(X ; R)$ is isomorphic to $\left(\omega_{2} ;<\right)$, contradicting Lemma 2.6 .

\section{REFERENCES}

[1] J. Brendle, S. Fuchino, and L. Soukup, Coloring ordinals by reals, to appear.

[2] E. K. van Douwen, The integers and topology, in Handbook of Set-Theoretic Topology, North-Holland, Amsterdam, 1984, pp. 111-167. MR 87f:54008

[3] M. Elekes, Linearly ordered families of Baire 1 functions, Real Analysis Exchange, 27 (2001/02), 49-63. 
[4] I. Juhász and K. Kunen, The power set of $\omega$, Fundamenta Mathematicae, Vol 170 (2001), 257-265.

[5] P. Komjáth, Ordered families of Baire-2-functions, Real Analysis Exchange, Vol 15 (1989-90), 442-444. MR 91d:26007

[6] K. Kunen, Inaccessibility Properties of Cardinals, Doctoral Dissertation, Stanford, 1968.

[7] K. Kuratowski, Topology, Vol. 1, Academic Press, 1966. MR 36:840

Department of Analysis, EÖtvös Loránd University, Budapest, Pázmány Péter sétány 1/C, 1117, HUNGARY

E-mail address: emarci@cs.elte.hu

Department of Mathematics, University of Wisconsin, Madison, Wisconsin 53706

E-mail address: kunen@math.wisc.edu

$U R L:$ http://www. math.wisc.edu ${ }^{\text {kunen }}$ 